\title{
ON THE SERIES OF PRIME RECIPROCALS
}

JAMES A. CLARKSON

Let $p_{n}$ be the $n$th prime. We give another proof of the

ThEOREM. The series $\sum_{n=1}^{\infty}\left(1 / p_{n}\right)$ diverges.

Proof. Assume the contrary, and fix $k$ so that

$$
\sum_{n=k+1}^{\infty}\left(1 / p_{n}\right)<1 / 2
$$

Let $Q=p_{1} p_{2} \cdots p_{k}$.

We consider now the sum $S(r)=\sum_{i=1}^{r}[1 /(1+i Q)]$, where $r$ is any positive integer. Since $1+i Q$ is prime to $Q$, all the prime factors of all these denominators are from a finite segment of primes which we call $P(r)$ :

$$
P(r)=\left\{p_{k+1}, p_{k+2}, \cdots, p_{m(r)}\right\} .
$$

Now let $S(r, j)$ stand for the sum of those terms in the sum $S(r)$ whose denominators $1+i Q$ have just $j$ (not assumed distinct) prime factors. Each such term has the form $1 / q_{1} q_{2} \cdots q_{j}$, with each $q_{t} \in P(r)$. But every such term occurs at least once in the expansion of $\left[\sum_{n=k+1}^{m(r)}\left(1 / p_{n}\right)\right]^{i}$, so by $(1) S(r, j)<1 / 2^{j}$. Thus for each $r$,

$$
S(r)=\sum_{j} S(r, j)<\sum_{j}\left(1 / 2^{j}\right)<1 .
$$

So $\sum_{i=1}^{\infty}[1 /(1+i Q)]$ converges, which in turn implies that the harmonic series does.

TuFts UNIVERsity

Received by the editors November 13, 1965. 